occlusion was 237 seconds. No increased thrombogenicity or vasospasm near the treated lesion was observed during the procedure.

Conclusions NGLEM, which is a DMSO free, non-adhesive bio-polymer may be used as an embolic material for the treatment of hemorrhagic stroke caused by cerebrovascular diseases.

Disclosures I. Yuki: 1; C; Research Grant: Institute for Clinical and Translational Science (ICTS) Pilot Award (NIH Director's Transformative Research Award initiative), Research Grant Supported by the MEXT (Ministry of Education, Culture, Sports, Science and Technology), Government of Japan. K. Ohkawa: 1; C; Research Grant Supported by the MEXT (Ministry of Education, Culture, Sports, Science and Technology), Government of Japan. 2; C; N/A. 3; C; N/A. 4; C; N/ A. 5; C; N/A. 6; C; N/A. F. Hsu: 1; C; Research Grant: Institute for Clinical and Translational Science (ICTS) Pilot Award. J. Xu: None. S. Li: None. S. Suzuki: 1; C; Research Grant: Institute for Clinical and Translational Science (ICTS) Pilot Award (NIH Director's Transformative Research Award initiative).

\section{E-009 A PROSPECTIVE, MULTICENTER REGISTRY FOR THE INVESTIGATION OF CLOT IN ISCHEMIC STROKE AND HEMATOMA EVACUATION: STUDY PROTOCOL FOR INSIGHT}

${ }^{1} \mathrm{C}$ Kellner*, ${ }^{2} \mathrm{~J}$ Fraser. ${ }^{1}$ Dept. of Neurosurgery, Mount Sinai Health System, New York, NY; ${ }^{2}$ Departments of Neurosurgery, Neurology, Radiology, and Neuroscience, University of Kentucky, Lexington, $K Y$

\subsection{6/neurintsurg-2020-SNIS.45}

Introduction/Purpose Multiple clinical studies have established the importance of effective clot evacuation on good clinical outcomes for acute ischemic and hemorrhagic stroke, and suggest that thrombus composition can impact treatment efficacy. Understanding clot composition and associated genomic, epigenomic, and proteomic signatures could provide insight into thrombus biology, etiology, and aid in prognosis. The primary objective of the INSIGHT study is to develop a 'multi-omic' blood clot signature to predict the etiology of the blood clot in patients with ischemic stroke or intracerebral hemorrhage $(\mathrm{ICH})$. Clots retrieved through either mechanical thrombectomy or endoscopic ICH aspiration will be analyzed with histology, RNA sequencing, and proteomics while contemporaneously collected peripheral blood samples will undergo single nucleotide polymorphism (SNP) analysis, RNA sequencing, and proteomic analysis. A secondary objective is to identify specimen characteristics that correlate with patient clinical presentation, procedural outcome, hospital length of stay, and 90-day functional outcome.

Materials and Methods INSIGHT is a prospective, multicenter registry for the collection and analysis of specimens collected from ischemic stroke thrombectomy procedures and minimally invasive interventions for intracerebral hematoma evacuation utilizing the Penumbra Aspiration Pump. The registry will enroll up to 1000 patients from up to 50 U.S. sites. Patients age $\geq 18$ years, treated frontline with the Penumbra System (for ischemic stroke patients eligible for mechanical thrombectomy) or the Artemis Neuro Evacuation device (for intracerebral hematoma evacuation in patients eligible for minimally invasive surgery), and with extracted thrombus/ embolus, will be included. Exclusion criteria includes current participation in a clinical trial that may confound the ability to capture clot and/or influence clot composition. Patient demographics, medical history, procedural information, and extracted clot and blood specimen will be collected. Followup assessments will occur at discharge and at 90 days postprocedure.

Results The estimated date for study completion is 2023.

Conclusion We report the design of the INSIGHT study, a prospective 'multi-omic' registry to analyze specimen collected during ischemic stroke thrombectomy and minimally invasive intracerebral hematoma evacuation.

Disclosures C. Kellner: None. J. Fraser: None.

\section{E-010 INVESTIGATION OF A NOVEL POLY(PROPYLENE GLYCOL) MATERIAL FOR USE AS A PROTEIN-RESISTANT, BIO-INERT IMPLANT}

${ }^{1} \mathrm{~W}$ Merritt*, ${ }^{2} \mathrm{~A}$ Koppisch, ${ }^{2} \mathrm{R}$ Kellar, ${ }^{1} \mathrm{~A}$ Ducruet, ${ }^{1} \mathrm{~T}$ Becker. ${ }^{1}$ Bioengineering Program, Northern Arizona University, Flagstaff, $A Z_{i}{ }^{2}$ Biology, Northern Arizona University, Flagstaff, $A Z$

\subsection{6/neurintsurg-2020-SNIS.46}

Introduction/Purpose A poly(propylene glycol)-based material (PPODA-QT) is currently being investigated as a liquid embolicfor the treatment of intracranial aneurysms. Initial biocompatibility results have shown that PPODA-QTis uniquely bioinert due to its ability to resist protein adsorption from contact with blood. Preliminaryanimal implant studies of PPODAQT in rabbits (1- and 3-month survivals) and canines (6month survival)have shown a lack of protein adsorption, lack of foreign body response, and minimal encapsulationaround the material. A protein-resistant, liquid-to-solid curing material could have expanded use as anon-fouling, protein-resistant coating for a variety of metal-based implants.

Materials and Methods The resistance to protein adsorption is quantified via protein depletion from the blood, as well asthrough analysis of desorbed proteins from sample surfaces via sodium dodecyl sulphate (SDS).Detection and quantification of proteins is performed via PPODA-QT samples $(n=12)$ were prepared andcured in $4 \mathrm{~mm}$ diameter cylindrical molds with a height of $10 \mathrm{~mm}$. PPODA-QT samples were eachimmersed in $1.5 \mathrm{~mL}$ of heparinized rabbit whole blood within a $2 \mathrm{~mL}$ polypropylene vial. Vials were placedon a shaker plate for 15 minutes to allow for ensure maximal interfacing between blood and thesamples. With the majority of protein adsorption happening within seconds, 15 minutes is sufficient forprotein adsorption. Positive controls $(n=4)$ were created by preparing $4 \mathrm{~mm}$ diameter and $10 \mathrm{~mm}$ thickpolyurethane cylinders and subjecting them to the same blood immersion procedure. Negative controls $(n=4)$ were created by filling vials with blood and no sample to give a baseline level for proteinadsorption onto the vials themselves. Proteins will be quantified via a protein quantitation assay thatutilizes a fluorescent dye to tag proteins for analysis with a spectrofluorometer with sensitivity as low as $10 \mathrm{ng} / \mathrm{mL}$.

Results PPODA-QT has been shown to exhibit exemplary protein-resistant properties as well as minimalencapsulation and inflammatory response when implanted while providing a relatively uniform surfacefor neointimal tissue growth across the device at the neck of the aneurysm. Comparison 